\title{
Bayesian exponential random graph modeling of whole-brain structural networks across lifespan
}

\author{
Michel Sinke \\ University Medical Center Utrecht, m.r.t.sinke@umcutrecht.nl \\ Rick Dijkhuizen \\ University Medical Center Utrecht \\ Alberto Caimo \\ Technological University Dublin, alberto.caimo@tudublin.ie
}

See next page for additional authors

Follow this and additional works at: https://arrow.tudublin.ie/scschmatart

Part of the Mathematics Commons, and the Medical Sciences Commons

\section{Recommended Citation}

Sinke, Michel; Dijkhuizen, Rick; Caimo, Alberto; Stam, Cornelis; and Otte, Willem, "Bayesian exponential random graph modeling of whole-brain structural networks across lifespan" (2016). Articles. 224.

https://arrow.tudublin.ie/scschmatart/224

This Article is brought to you for free and open access by the School of Mathematics at ARROW@TU Dublin. It has been accepted for inclusion in Articles by an authorized administrator of ARROW@TU Dublin. For more information, please contact arrow.admin@tudublin.ie, aisling.coyne@tudublin.ie,gerard.connolly@tudublin.ie.

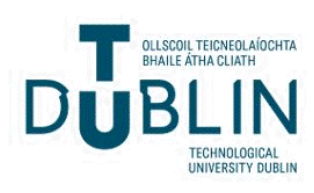




\section{Authors}

Michel Sinke, Rick Dijkhuizen, Alberto Caimo, Cornelis Stam, and Willem Otte

This article is available at ARROW@TU Dublin: https://arrow.tudublin.ie/scschmatart/224 


\title{
Bayesian exponential random graph modeling of whole-brain structural networks across lifespan
}

\author{
Michel R.T. Sinke ${ }^{\mathrm{a}, *}$, Rick M. Dijkhuizen ${ }^{\mathrm{a}}$, Alberto Caimo ${ }^{\mathrm{b}}$, Cornelis J. Stam ${ }^{\mathrm{c}}$, Willem M. Otte ${ }^{\mathrm{a}, \mathrm{d}, \mathrm{e}}$ \\ a Biomedical MR Imaging and Spectroscopy Group, Center for Image Sciences, University Medical Center Utrecht, Utrecht, The Netherlands \\ b School of Mathematical Sciences, Dublin Institute of Technology, Dublin, Ireland \\ c Department of Clinical Neurophysiology, Neuroscience Campus Amsterdam, VU University Medical Center, Amsterdam, The Netherlands \\ d Department of Pediatric Neurology, Brain Center Rudolf Magnus, University Medical Center Utrecht, Utrecht, The Netherlands \\ e Stichting Epilepsie Instellingen Nederland (SEIN), Heemstede, The Netherlands
}

\section{A R T I C L E I N F O}

\section{Article history:}

Received 18 February 2016

Revised 1 April 2016

Accepted 26 April 2016

Available online 28 April 2016

\section{Keywords:}

Bayesian statistics

$\mathrm{P}^{*}$ model

Connectome

Aging

Diffusion tensor imaging

Tractography

Generative network analysis

\begin{abstract}
A B S T R A C T
Descriptive neural network analyses have provided important insights into the organization of structural and functional networks in the human brain. However, these analyses have limitations for inter-subject or between-group comparisons in which network sizes and edge densities may differ, such as in studies on neurodevelopment or brain diseases. Furthermore, descriptive neural network analyses lack an appropriate generic null model and a unifying framework. These issues may be solved with an alternative framework based on a Bayesian generative modeling approach, i.e. Bayesian exponential random graph modeling (ERGM), which explains an observed network by the joint contribution of local network structures or features (for which we chose neurobiologically meaningful constructs such as connectedness, local clustering or global efficiency). We aimed to identify how these local network structures (or features) are evolving across the life-span, and how sensitive these features are to random and targeted lesions. To that aim we applied Bayesian exponential random graph modeling on structural networks derived from whole-brain diffusion tensor imaging-based tractography of 382 healthy adult subjects (age range: 20.2-86.2 years), with and without lesion simulations. Networks were successfully generated from four local network structures that resulted in excellent goodness-of-fit, i.e. measures of connectedness, local clustering, global efficiency and intrahemispheric connectivity. We found that local structures (i.e. connectedness, local clustering and global efficiency), which give rise to the global network topology, were stable even after lesion simulations across the lifespan, in contrast to overall descriptive network changes - e.g. lower network density and higher clustering - during aging, and despite clear effects of hub damage on network topologies. Our study demonstrates the potential of Bayesian generative modeling to characterize the underlying network structures that drive the brain's global network topology at different developmental stages and/or under pathological conditions.
\end{abstract}

(c) 2016 Elsevier Inc. All rights reserved.

\section{Introduction}

Aging is a major risk factor of prevalent diseases in society, including neurodegenerative disorders such as Alzheimer's and Parkinson's disease (Collier and Kordower, 2012; Niccoli and Partridge, 2012). During aging the human brain is subject to structural and functional changes that can cause behavioral problems and cognitive decline (e.g. reduced executive functioning or memory impairment). However, many elderly people do not suffer from behavioral and cognitive problems and are functioning well, despite structural and functional changes in brain networks. Therefore it is important to understand to what extent specific

\footnotetext{
* Corresponding author at: Biomedical MR Imaging and Spectroscopy Group, Center for Image Sciences, University Medical Center Utrecht, Yalelaan 2, 3584 CM Utrecht, The Netherlands.

E-mail address: m.r.t.sinke@umcutrecht.nl (M.R.T. Sinke).
}

changes in brain connectivity across the lifespan contribute to increased risk and development of age-related neurological disorders, even in the absence of significant brain pathology (Burke and Barnes, 2006).

Graph analysis has proven to be an elegant tool to assess topological aspects of structural and functional connectivity in the brain (Bullmore and Sporns, 2009). Graph analysis describes the brain as a set of nodes, representing neural elements, linked by edges, representing some measure of structural, functional or causal interaction between the nodes. Many studies have successfully applied graph analysis to capture network topologies with either individual or aggregated node metrics (e.g. the average shortest path length, maximum betweenness centrality or overall clustering coefficient) (Bullmore and Sporns, 2009) and/or network properties such as small-worldness, rich club connectedness (Bullmore and Sporns, 2012; Cao et al., 2014) and modularity (Rubinov and Sporns, 2010). In the past decade, multiple studies have shown that normal aging is associated with substantial alterations in 
structural (Betzel et al., 2014; Dennis et al., 2013; Gong et al., 2009; Hagmann et al., 2010; Lim et al., 2015; Montembeault et al., 2012; Otte et al., 2015; Wu et al., 2012; Zhu et al., 2012) and functional (Achard and Bullmore, 2007; Andrews-Hanna et al., 2007; Betzel et al., 2014; Meier et al., 2012; Meunier et al., 2009; Nathan Spreng and Schacter, 2012; Wang et al., 2012) brain networks. Some of these studies focused on specific age categories: childhood to adulthood (Dennis et al., 2013; Hagmann et al., 2010) or young and older adults (e.g. (Meunier et al., 2009; Zhu et al., 2012)). From childhood to adulthood a decrease in path length and clustering, and increase in efficiency have been observed (Dennis et al., 2013; Hagmann et al., 2010) which may differ between the hemispheres (Dennis et al., 2013). Other studies have shown a higher local clustering and lower global efficiency in older adults compared to younger adults (Zhu et al., 2012), where modularity decreases across networks (Meunier et al., 2009). In line with these findings several studies have shown inverted-U shaped global efficiency across lifespan (Otte et al., 2015; Wu et al., 2012). Functional connectivity assessment has revealed increased integration and decreased randomness, whereas connectivity decreased significantly during adulthood (Smit et al., 2016). Despite these significant network changes, throughout development brain networks largely maintain small-world properties, modularity and stable hub-regions (Dennis et al., 2013; Gong et al., 2009; Hagmann et al., 2007). In general, the aging brain can be characterized by reduced centrality of hub regions with a decrease in global efficiency and an increase in local network clustering. Similar changes in hub regions and subsequent effects on global efficiency have also been characterized in various neurological disorders (Crossley et al., 2014; Stam, 2014).

Despite the popularity of descriptive graph analysis, it has nontrivial intrinsic limitations, particularly for intersubject or betweengroup comparisons where networks have different sizes, densities and degree distributions (Fornito et al., 2013; van Wijk et al., 2010). The most commonly used node metrics, like the clustering coefficient and path length, are highly dependent on the total number of connections and the average degree of a network (Stam et al., 2014; van Wijk et al., 2010). This hampers comparability of brain network topology across the human lifespan, as network densities substantially differ between ages (Dennis et al., 2013; Gong et al., 2009; Hagmann et al., 2010), which may be explained by changes in the integrity of connecting fibers or the cortical density of neurons (Salat, 2011; Westlye et al., 2010).

A second limitation is the lack of an appropriate generic null model to test the significance of a particular network measure against. A frequently used null model is a network with randomly shuffled edges that shares basic characteristics with the measured network, like degree distribution, size and density. Different network metrics require distinct null models if compared between networks (e.g., networks with asymmetric degree distributions cannot be explained by the Watts-Strogatz small-world network null model but require the Barabási-Albert scalefree model) (Fornito et al., 2013).

A third limitation of graph analysis is the type I error inflation if multiple network nodes are compared within the same brain, or if different network metrics are calculated from a single network.

Fourthly, graph analysis consists of univariable comparisons (i.e., network metrics are determined independent from each other) due to lack of a unifying framework (Telesford et al., 2011). However, many metrics are highly correlated and non-exclusive (Bounova and De Weck, 2012; Meghanathan, 2015).

A promising alternative analysis approach, which may in theory overcome the abovementioned limitations in descriptive graph analysis, is the framework of generative modeling (Fornito et al., 2013; Klimm et al., 2014), which aims to condense a complex network topology into a parsimonious description (i.e. mathematical equation). Growth models are a relatively well known class of generative models. They involve growing of artificial networks via addition of nodes and edges and rewiring of existing edges according to pre-specified mechanisms, and comparing topologies between these artificially grown and observed networks. Relatively simple growing mechanisms (i.e., the mathematical local structures) provide a generative model that allows growing of networks that closely resemble observed brain networks. A recent successful example is a growth model with two local structures: a combined distance penalty based on the cost of maintaining long-range connections and a topological term that favors links between regions sharing similar input (Vertes et al., 2012). Similar principles have been successfully applied by other recent studies on neural networks (Betzel et al., 2015; Goni et al., 2014). However, unambiguous determination of distances between non-connecting pairs of network nodes required for distance penalties - is difficult.

Another recent and powerful class of generative models are the exponential random graph models. Their usefulness has been emphasized in social network studies (Robins et al., 2007b), but they may have equal potential for neuronal networks (Simpson et al., 2011). Until recently, exponential random graph models have been difficult to handle from a statistical point of view, due to the intractability of the normalizing constant and the problem of model degeneracy (Handcock, 2003), which has limited their applicability. The recent presentation of a Bayesian inference framework, using adaptive Markov chain Monte Carlo approaches to fit exponential random graph models, mitigated the issue of model degeneracy and significantly improved fitting performance (Caimo and Friel, 2011). Exponential random graph models are able to explore multiple local network features (e.g. connectedness, local clustering or global efficiency) simultaneously and assess how these local features give rise to the global network topology, thereby taking into account their mutual dependencies (note that the term 'local' is defined from a topological and not from a physical perspective, i.e. local clustering does not necessary imply physical proximity of involved nodes). In addition, exponential random graph models inherently account for bias due to density differences (van Wijk et al., 2010). More technically, the models capture the joint probability of a (global) network $G$, governed by $\vartheta$, a set of network parameters (e.g. local clustering, edges) of a postulated generative process. If $\vartheta$ is estimated well, synthetic networks - which are structurally similar to $G$ - may be drawn from a probability distribution $P(G \mid \vartheta)$. Exponential random graph modeling may thus also be considered as a mathematical framework to condense the (global) topological information of a network into a limited set of parameters (i.e. the local network structures or features). This mathematical description theoretically provides: $i$ ) compression of the observed network data into a basic equation, ii) capturing of the most relevant patterns within the observed network, iii) generalization from the observed network to unobserved networks of the same type, iv) generalization across network sizes, and $v$ ) prediction of network topologies.

Exponential random graph models may provide unambiguous answers to fundamental questions related to brain-wide network organization and changes across lifespan, such as: how do local network features (i.e. neurobiologically meaningful constructs such as local clustering, connectedness and global efficiency) simultaneously give rise to (i.e. explain) the global network topology, and what is the relative significance (i.e. contribution) of those local structures during development and aging? Will there still be changes across lifespan in brain global efficiency or local clustering, if confounding effects such as correlations between metrics and decreased network density with increased age, are effectively taken into account? In fact, surprisingly little is known on how local network features simultaneously shape the global network characteristics so commonly reported in descriptive graph analysis studies. Furthermore, it is unknown to what extent local structures are affected by specific network damage in brain injuries and pathologies. For example, does damage to central hub regions result in distinct local features (such as brain network clustering or connectedness) as compared to diffuse network damage, and does this differ across the lifespan? 
Our study objective was to characterize the relative contributions of local network structures that might explain the configuration of structural whole-brain networks across the human lifespan. To that aim we fitted Bayesian exponential random graph models on structural networks, obtained from diffusion tensor imaging (DTI) data from 382 healthy subjects in the age range of 20 to 86 years. As these models are based on probability estimates, we were able to directly compare the optimally fitted parameters that explain the network topology between different age groups. Furthermore we evaluated in simulations the consequences of hub lesioning and diffuse network damage on the local structures across the human lifespan. We hypothesized that Bayesian exponential random graph modeling could characterize the observed networks with a limited set of network parameters, and would allow generation of synthetic networks that are topologically similar to the observed networks. Based on findings from descriptive graph analysis in healthy subjects (Otte et al., 2015) we expected: $i$ ) a decrease in contribution of global network efficiency structures and density (e.g. total number of edges) with age, ii) an increase in local clustering structures with age, and iii) significant changes in relative contributions of local substructures when hub nodes are damaged.

\section{Material and methods}

\subsection{Dataset}

Standardized high quality, DTI and $\mathrm{T}_{1}$-weighted datasets were obtained from the freely available 'Information extraction from images' database (http://biomedic.doc.ic.ac.uk/brain-development/). We included scans from 382 healthy adults recruited from the general United Kingdom population. The age range was 20.2 to 86.2 years. This DTI dataset has been analyzed previously to characterize the backbone of the structural whole-brain network across the lifespan (Otte et al., 2015).

Subjects were scanned once with a 1.5T (205 subjects, Philips Medical Systems Gyroscan Intera) or 3T (177 subjects, Philips Medical Systems Intera). Ethical approval was given by the Thames Valley multicenter ethics committee. Images were acquired using a singleshot echo-planar (EPI) DTI sequence. Imaging parameters were: 15 diffusion-weighted images with $\mathrm{b}=1000 \mathrm{~s} / \mathrm{mm}^{2}, 2$ images without diffusion-weighting, 56 axial slices, $2.35 \mathrm{~mm}$ slice-thickness, $128 \times 128$ acquisition matrix, $1.75 \times 1.75 \mathrm{~mm}$ voxels; repetition time (TR)/echo time (TE) $9.1 \mathrm{~s} / 80 \mathrm{~ms}$ (at $1.5 \mathrm{~T}$ ) or $11.9 \mathrm{~s} / 51 \mathrm{~ms}$ (at 3T). In addition, $\mathrm{T}_{1}$-weighted images were acquired using a spin-echo sequence with the following parameters: $8^{\circ}$ flip angle, TR/TE 9.6/4.6 ms (at 1.5T) or TR/TE $9.8 / 4.6 \mathrm{~ms}$ (at 3T), 150 axial slices, $1.2 \mathrm{~mm}$ slicethickness, $256 \times 256$ acquisition matrix, $0.94 \times 0.94 \mathrm{~mm}$ voxels.

\subsection{Image processing}

All diffusion-weighted images were corrected for subject motion using FLIRT followed by B-matrix adjustment using the affine registration parameters. Within-subject diffusion-weighted and $\mathrm{T}_{1}$-weighted images were non-rigidly aligned, and subsequently registered to a $\mathrm{T}_{1^{-}}$ weighted reference image that was matched to the Harvard-Oxford atlas (Desikan et al., 2006). Using the 50\% probability threshold Harvard-Oxford cortical parcellation masks, we partitioned each hemisphere in 48 cortical network regions, i.e. 96 network regions bilaterally, based on standard anatomical boundaries.

Analysis of DTI data was performed using the Diffusion Toolkit (Wang et al., 2007). Diffusion images were resampled on an isotropic $1.75 \mathrm{~mm}$ grid, and fractional anisotropy (FA) maps were calculated based on voxel-wise estimates of the diffusion tensor. Whole-brain tractography was obtained from seeds in each voxel according to FA $>0.2$ using the interpolated streamline algorithm implementation (Euler integration method with step size of $0.5 \mathrm{~mm}$ ), with streamlines originating from ten random seeds in each voxel, a maximal $70^{\circ}$ angle threshold and minimal FA threshold of 0.2 (DtiStudio version 0.6). The algorithm is based on the Fiber Assignment by Continuous Tracking (FACT) approach, by which tracking is performed using a continuous coordinate system rather than a discrete voxel grid. Structural connections between any pair of cortical network regions were identified from streamlines that had end points in both regions.

The image processing pipeline is schematically illustrated in Fig. 1.

\subsection{Construction of whole-brain structural networks}

For each of the 382 sets of whole-brain structural connections we constructed a binary undirected network, described by the graph $G=$ $(N, E)$ where $\mathrm{N}$ is the set of 96 bilateral cortical network regions and $E$ is the set of edges $e_{i j}$ in the $N \times N$ adjacency matrix, with $e_{i j}$ set to 1 if region pairs were connected with one or more streamlines, or 0 otherwise. No self-connections were allowed.

Participants were divided into different age-categories: 20-34, 35$50,51-70$ and $\& 70$ years. For each age category individual graphs were summed and divided by the total number of participants within each age category, resulting in an average (weighted) connectivity matrix that shows the proportion of participants having a specific connection for each node pair (Fig. S1). Edges in the age-category graphs were defined as present if $>35 \%$ of subjects within that age range contained an edge in their individual network (Fig. S2). By using this 35\% threshold we included at least $25 \%$ of prevalent connections over all age categories. All subsequent analyses were based on these binary age-category graphs.

The 'hubness' of individual nodes - required for the network damage simulations - was calculated as the betweenness centrality and ranked from high to low (i.e., central to peripheral centrality) (Rubinov and Sporns, 2010). Hub nodes have a high betweenness centrality, i.e. a relatively high number of shortest paths passing through the network nodes. The betweenness centrality of node $i$ is defined as

$$
b c_{i}=\frac{1}{(n-1)(n-2)} \sum_{j \neq k, k \neq i, j \neq i}^{n} \frac{g_{j k}(i)}{g_{j k}}
$$

where $g_{j k}$ is the shortest path between a pair of other nodes and $g_{j k}(i)$ is the number of those node paths that pass through node $i$.

\subsection{Bayesian exponential random graph modeling}

Exponential random graph models constitute a broad class of network models that assume that the topological structure of an observed network $x_{o b s}$ can be explained in terms of the relative contribution of a set of mutual dependent local structures or features $g(x)$ (e.g. clustering coefficient, path length, location in the brain). The models require a defined network distribution $\chi(n)$ that contains all possible binary networks with size $n$. In our study, $n$ was 96 , the number of atlas regions. A network can then be represented by a random variable $\boldsymbol{X}$. A realization of $\boldsymbol{X}$ is denoted by $x=\left\{x_{i j}\right\}$. An observed network (e.g. one of the four age-category graphs) $x_{o b s}$ is regarded as just one realization from the set of all possible networks $\boldsymbol{X}$ with similar network topology. In other words, $x_{o b s}$ is assumed to be the outcome of some stochastic process. The goal of exponential random graph models is to propose a plausible model for this stochastic process (Frank and Strauss, 1986; Pattison and Wasserman, 1996). More formally, exponential random graph models are a particular class of discrete linear exponential families that represent the probability distribution of $\boldsymbol{X}$ as:

$\pi(\mathbf{X}=x \mid \theta)=\frac{\exp \left\{\theta^{t} g(x)\right\}}{z(\theta)}$

where $\theta \in \Theta \subset \mathfrak{R}^{q}$ is the vector of model coefficients (that has to be estimated) related to $g(\boldsymbol{x})$ which quantifies the relative contribution (i.e. significance) of each local network feature (e.g. local clustering, global 


\section{Whole-brain tractography $(n=382)$}
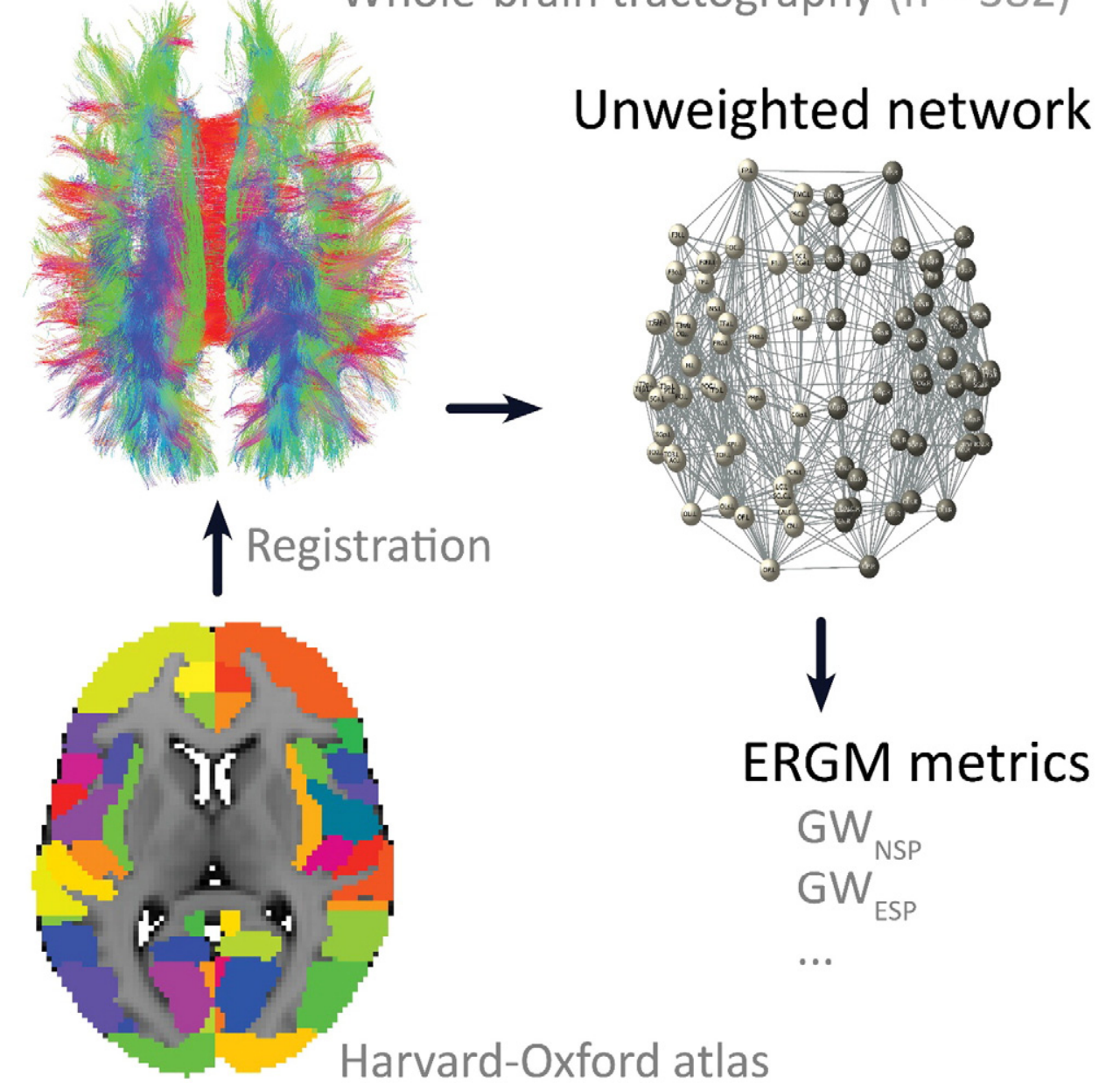

ERGM metrics

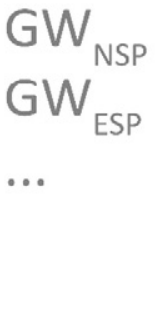

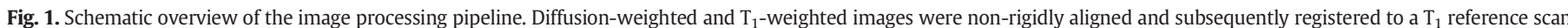

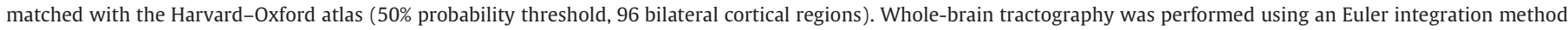

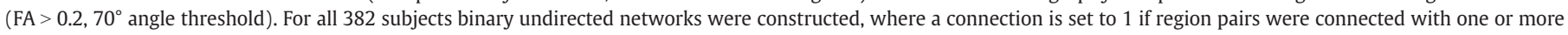
streamlines, or 0 otherwise. Graphs were averaged into four age-category networks. ERGM metrics (e.g. GW ESP, $_{\text {, GW }}$ NP) were calculated from these networks.

efficiency) in explaining the global structure of $x_{o b s}$, thereby taking into account the contribution of other network features in the model. The $\boldsymbol{\theta}$ represents the change in log-odds of an edge existing for each unit increase for a specific explanatory parameter (i.e. clustering) in the model. If $\boldsymbol{\theta}$ is large and positive for this specific local structure, then this metric plays an important role in explaining the global network structure (i.e. local clustering contributes more to the network than you would expect by chance). If $\boldsymbol{\theta}$ is large and negative, then this metric also plays an important role in explaining the overall network topology, but is less important (i.e. there is less local clustering) than expected by chance. Furthermore, $z(\theta)=\sum_{\chi \in \chi} \exp \left\{\theta^{t} g(\mathbf{x})\right\}$ is the normalizing constant which is generated over the entire network space $\chi(n)$ to ensure that (1) is a proper probability distribution. Since $\chi(n)$ consists of $2^{n \times n}$ possible networks, the normalizing constant $z(\boldsymbol{\theta})$ (i.e. ensures that the probabilities sum to one) is intractable. To avoid the need to calculate $z(\boldsymbol{\theta})$, a pseudolikelihood estimation method has been proposed (Strauss and Ikeda, 1990). Although fitting exponential random graphs using this pseudolikelihood estimation is computationally relatively easy, the properties of the estimator are not well understood and the estimated $\boldsymbol{\theta}$ are known to be inaccurate in many cases (Robins et al., 2007a). An alternative and more straightforward method to fit exponential random graph models is by means of Monte Carlo maximum likelihood estimation (Hunter and Handcock, 2006). This form of estimation simulates a distribution of random networks from a starting set of model parameters, $\boldsymbol{\theta}_{0}$, and subsequently refines the parameter values by comparing the distribution of networks against the measured network $x_{o b s}$. A crucial aspect of this estimation is a proper choice of $\boldsymbol{\theta}_{0}$. Poorly chosen initial values may lead to non-convergence or degenerative probability distributions, and thus result in unreasonable estimates of $\boldsymbol{\theta}$. For details on this issue see (Handcock, 2003) and (Fienberg et al., 2008).

Recently a Bayesian solution for this issue of proper initialization of $\boldsymbol{\theta}$ was proposed and successfully tested in the area of social network modeling (Caimo and Friel, 2011; Caimo and Mira, 2014). In this method a prior distribution $\pi(\boldsymbol{\theta})$ is assigned to $\boldsymbol{\theta}$. The posterior distribution $\pi(\theta \mid x) \propto \pi(\mathbf{x} \mid \theta) \pi(\theta)$ is subsequently solved with a Monte Carlo maximum likelihood method based on the exchange algorithm (Murray et al., 2006). This Bayesian framework has been shown to perform very well, and was used in the current study as the method of choice in fitting exponential random graph models.

\subsection{Local structure definitions}

A large set of local structures are provided in the literature to use with exponential random graph models (Hunter et al., 2009). Two previous studies (Simpson et al., 2011, 2012) included local structures that resemble most of the descriptive network characteristics used in classical graph analysis studies, i.e. connectedness, local clustering and global efficiency (Bullmore and Sporns, 2009; Morris et al., 2008; Rubinov and 
Sporns, 2010). These studies showed that three local structures or features, i.e. the non-edgewise share partners, edgewise shared partners and edges, contributed significantly in most functional brain networks (Simpson et al., 2011, 2012). Although we investigated structural rather than functional networks, we used the same structures for our analyses; first, because many (classical) graph analysis studies have also used similar graph metrics (e.g. clustering coefficient, path length, modularity) to describe both functional and structural networks; second, because there is a strong correlation between structural and functional networks; and third, because it has been shown that structural connections are predictive of functional connections (Hagmann et al., 2007; Honey et al., 2009; Sporns, 2010). In addition, we included a local structure based on the hemispheric attribution of a node (i.e. whether a node tended to make more connections within or between hemispheres) as the white matter connectivity within hemispheres is found to be higher in comparison to the connectivity between hemispheres (Hagmann et al., 2008). Fig. 2 shows a graphical representations of local structures used in network generation. These were defined as:

The number of edges

$s(\mathbf{x})=\sum_{i<j} x_{i j}$

A measure of connectedness compatible with exponential random graph modeling, i.e. an increase in the edge coefficient means an increase in number of connections, or a higher network density.

The geometrically weighted edgewise shared partner (GWESP)

$w\left(\mathbf{x}, \phi_{v}\right)=e^{\phi \mathrm{w}} \sum_{k=1}^{n-2}\left\{1-\left(1-e^{-\phi \mathrm{w}}\right)^{k}\right\} E P_{k}(\mathbf{x})$

With $E P_{k}(x)$ defined as the number of pairs $\{i, j\}$ such that $x_{i j}=1$ and $i$ and $j$ have exactly $k$ common neighbors, which is a measure of local clustering compatible with exponential random graph modeling. An increase in $\mathrm{GW}_{\mathrm{ESP}}$ coefficient means more local clustering (segregation). In other words, two connected nodes (brain regions) tend to connect to similar other brain regions (i.e. shared partners).

The geometrically weighted non-edgewise shared partner (GWNSP)

$p\left(\mathbf{x}, \phi_{p}\right)=e^{\phi \mathrm{p}} \sum_{k=1}^{n-2}\left\{1-\left(1-e^{-\phi \mathrm{p}}\right)^{k}\right\} N P_{k}(\mathbf{x})$

With $N P_{k}(x)$ defined as the number of pairs $\{i, j\}$ such that $x_{i j}=0$ and $i$ and $j$ have exactly $k$ common neighbors, which is a measure of global efficiency compatible with exponential random graph modeling. An increase in $\mathrm{GW}_{\mathrm{NSP}}$ coefficient indicates there is more global efficiency (integration); an increase in non-connected nodes, sharing one or more connections to the same brain regions (i.e. shared partners). This could be more distant brain regions which are indirectly connected (i.e. via a shared partner), or a so-called connector-hub, connecting two network modules (Bullmore and Sporns, 2009).

The parameter $\phi$ in Eqs. (3) and (4) dampens the effect of large changes in the statistics of higher $k$ and was in this study fixed to 0.75 so that the model remains a regular exponential random graph model (Hunter and Handcock, 2006).

The hemispheric nodematch

A binary nodematch-local structure, called hemispheric nodematch, captured whether two nodes belong to the same hemisphere (left or right).

$h(\mathbf{x})=\sum_{i<j} \mathbf{x}_{i j} \mathbf{x}_{j i}: m_{i}=m_{j}$

With $m$ as the hemisphere membership.

\subsection{Summary estimates}

The Bayesian exponential random graph models provide full probability density estimates. We summarized the posterior probability density estimates of the $\boldsymbol{\theta}$ coefficients - which correspond with the local structures - as the mean and 95\% credibility intervals.

\subsection{Goodness-of-fit}

We assessed the data goodness-of-fit to the posterior model by comparing the network data $\mathbf{y}$ by means of a set of network simulations $\left(\boldsymbol{y}_{1}\right.$, $\left.\boldsymbol{y}_{2}, \ldots \boldsymbol{y}_{\boldsymbol{S}}\right)$ from $S$ independent realizations $\left(\boldsymbol{\theta}_{1}, \boldsymbol{\theta}_{2}, \ldots \boldsymbol{\theta}_{\boldsymbol{S}}\right)$ of the posterior density estimate. We then compared the observed network with the simulated network based on three global descriptive network characteristics: degree (connectivity), geodic distance (shortest path) and edge-wise shared partners (clustering) from the Bergm package (Caimo and Friel, 2014). Additionally, we implemented the triad census (i.e., subset of motifs), that determines the contributions, as a probability, of one, two or three connections between all possible node triples (Hunter et al., 2009; Morris et al., 2008), and compared these contributions between the observed and simulated networks.

\subsection{Effect of simulated network damage}

We estimated the effect of network damage on the local structures/ features by simulating lesions in our data. First we simulated lesions by randomly eliminating $5 \%$ to $25 \%$ of the nodes and connecting edges in steps of $5 \%$. We applied the same procedure for the hub-nodes, removing
A

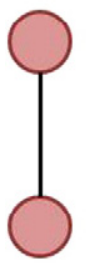

B

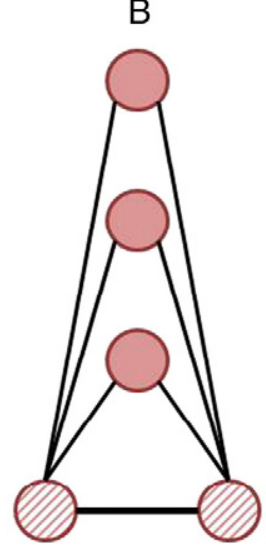

$\mathrm{C}$

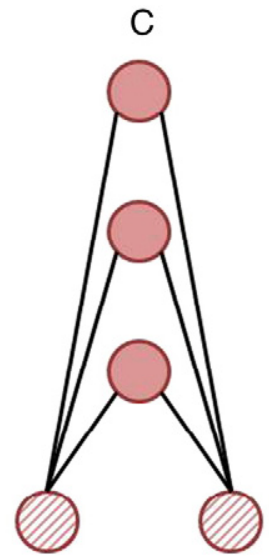

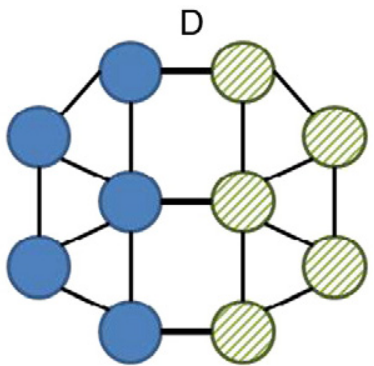

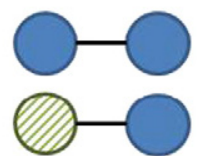

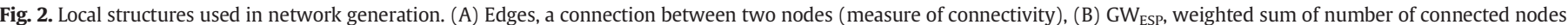

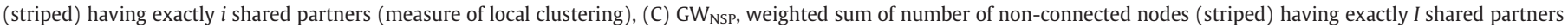

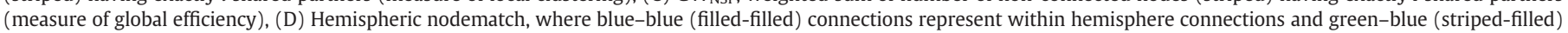
connections represent between hemisphere connections. 
Table 1

Log transformed Bayes factors and their interpretation in the comparison of a baseline and alternative model (Raftery, 1995).

\begin{tabular}{ll}
\hline $\log$ (Bayes factor) & Interpretation \\
\hline$<-5$ & Very strong support for baseline model \\
-5 to -3 & Strong support for baseline model \\
-3 to -1 & Positive support for baseline model \\
1 to 0 & Weak support for baseline model \\
0 & No support for baseline model \\
0 to 1 & Weak support for alternative model \\
1 to 3 & Positive support for alternative model \\
3 to 5 & Strong support for alternative \\
$>5$ & Very strong support for alternative model \\
\hline
\end{tabular}

increasing percentages of nodes with the highest betweenness centrality. We evaluated the consequences of random-node and hub-node damage on the local structure parameters (e.g. edges, GW $\mathrm{ESP}_{\mathrm{ES}} \mathrm{GW}_{\mathrm{NSP}}$, and hemispheric nodematch). The effect of simulated damage between percentages of eliminated nodes was quantified with $95 \%$ credibility intervals obtained from the difference between the posterior distributions.
Vulnerability to hub-node elimination - defined as a change in local substructure parameter between $0 \%$ and $25 \%$ eliminated nodes - was subsequently compared between age categories based on Bayes factors obtained from a Bayesian equivalent of an independent two sample t-test (Rouder et al., 2009). Bayes factors give the ratio of model likelihoods, thereby providing which model (i.e., the presence or absence of a difference in the parameter change) is supported (i.e., more likely to occur) (Caimo and Friel, 2011). The interpretation of Bayes factors is given in Table 1.

\subsection{Statistical analyses}

All network analysis, statistical modeling and visualization were performed in R (http://www.r-project.org/) using the open-source packages igraph, Bergm, Bayes Factor, network and ggplot2.

\section{Results}

\subsection{Network matrices and model parameters}

We generated group-based networks for each age category, which are depicted in Fig. 3 as network graph representations (for binary

\section{Age 20-34}

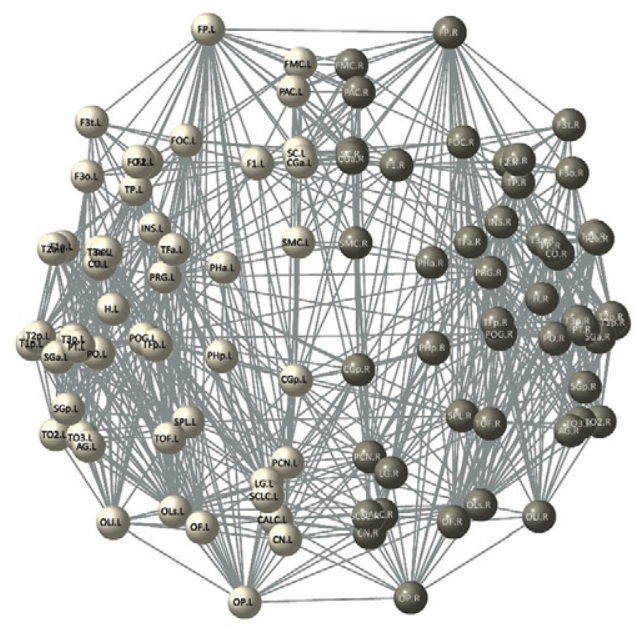

Age $20-34$ vs Age $\mathbf{5 1 - 7 0}$

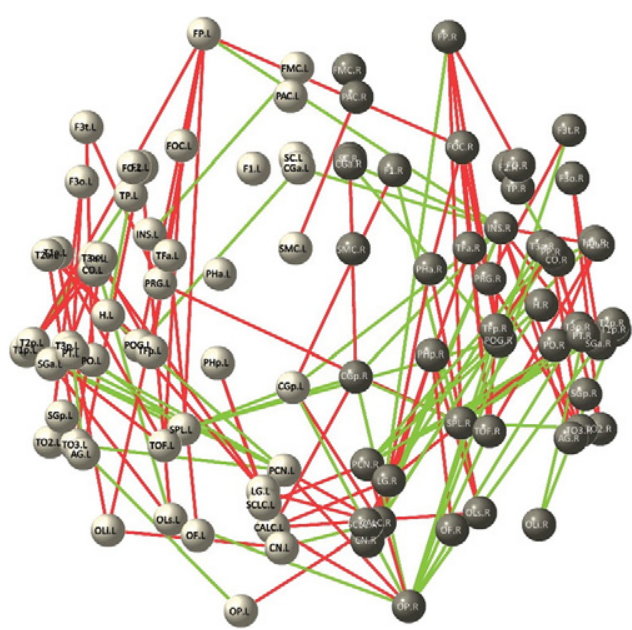

Age $\mathbf{2 0 - 3 4}$ vs Age $\mathbf{3 5 - 5 0}$

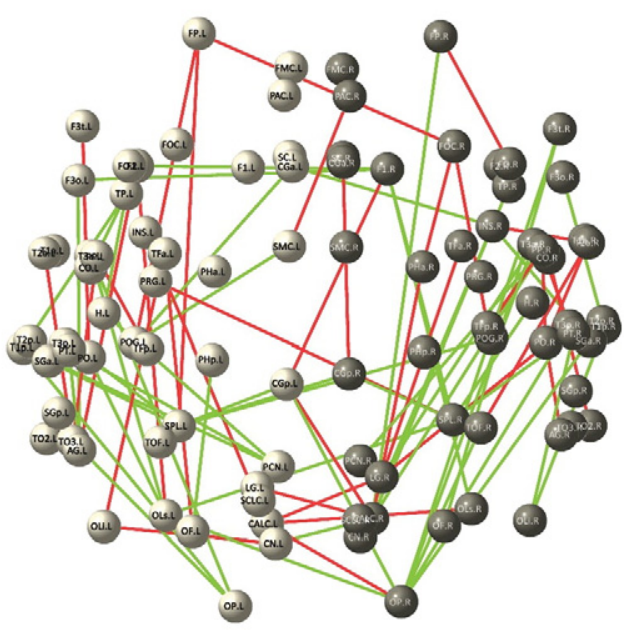

Age $20-34$ vs Age >70

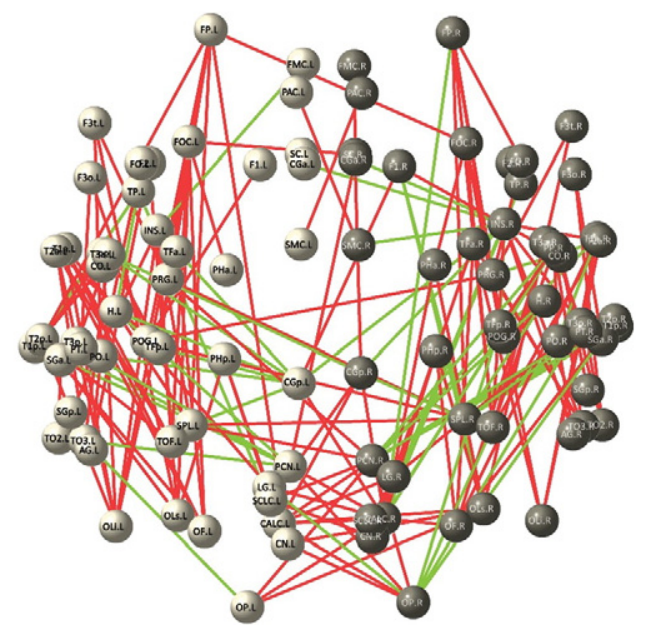

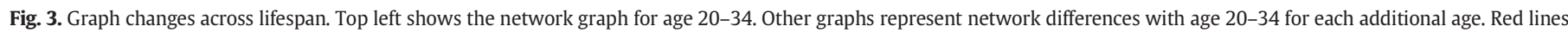

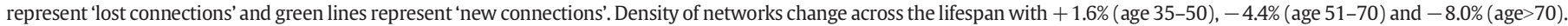


Table 2

Mean model parameter coefficients and 95\% credible (i.e. posterior probability) intervals for each age category.

\begin{tabular}{|c|c|c|c|c|}
\hline \multirow[b]{2}{*}{ Age category } & \multicolumn{4}{|c|}{ Model parameter coefficients } \\
\hline & Edges & $\mathrm{GW}_{\mathrm{ESP}}$ & $\mathrm{GW}_{\mathrm{NSP}}$ & Hemispheric nodematch \\
\hline $20-34$ & $-2.38(-3.45$ to -1.31$)$ & $1.52(1.04-1.99)$ & $-0.27(-0.34$ to -0.20$)$ & $-0.68(-1.32$ to -0.05$)$ \\
\hline $35-50$ & $-2.36(-3.41$ to -1.32$)$ & $1.49(1.02-1.96)$ & $-0.27(-0.34$ to -0.20$)$ & $-0.72(-1.35$ to -0.09$)$ \\
\hline $51-70$ & $-2.41(-3.43$ to -1.39$)$ & $1.53(1.06-1.99)$ & $-0.27(-0.34$ to -0.20$)$ & $-0.75(-1.36$ to -0.15$)$ \\
\hline$>70$ & $-2.61(-3.58$ to -1.64$)$ & $1.63(1.20-2.06)$ & $-0.25(-0.31$ to -0.18$)$ & $-0.89(-1.53$ to -0.24$)$ \\
\hline
\end{tabular}

adjacency matrices, see Fig. S3). Exponential random graph models were estimated for each age category with the four local network structures edges, $\mathrm{GW}_{\mathrm{NSP}}, \mathrm{GW}_{\mathrm{ESP}}$ and hemispheric nodematch. All local structures were found to contribute significantly to the global network topology. Parameter coefficients and corresponding credible intervals are given in Table 2. Goodness-of-fit measures showed that the simulated networks matched well with the observed networks for all age categories. The goodness-of-fit metrics (i.e. degree, geodesic distance and edge-wise shared partners) and triad census contributions of simulated networks almost completely matched with the metrics of the observed networks, as seen in Fig. 4 and Fig. S4, respectively.

\subsection{Global and local network features}

Clear alterations in network topology were found in the older age categories compared with the youngest age category of 20 to 34 years (Fig. 3). Across the lifespan new connections are found and other connections are lost (represented by green and red lines respectively),
Age 20-34

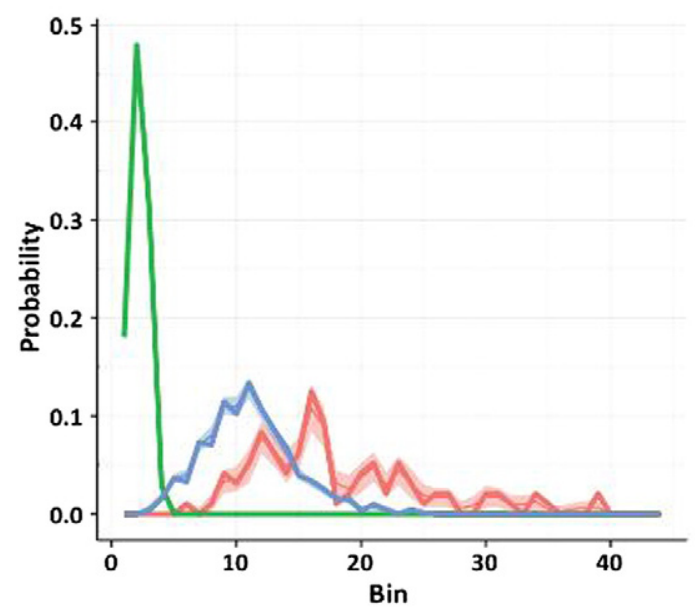

Age 51-70

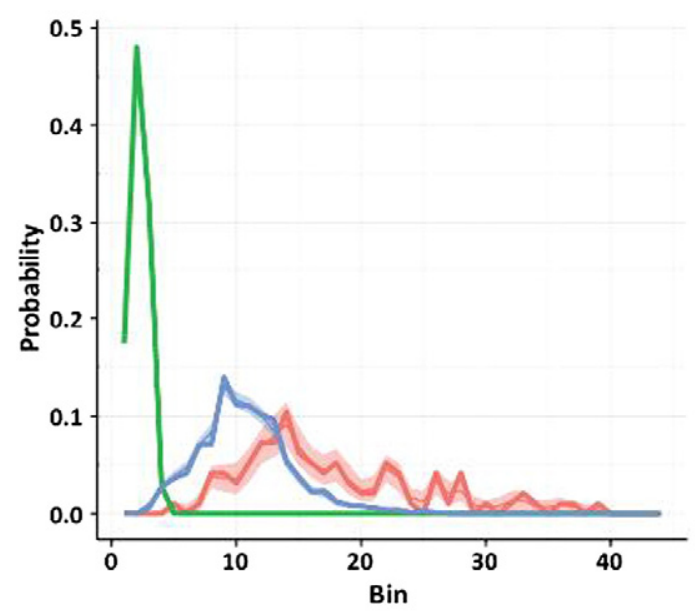

Age 35-50

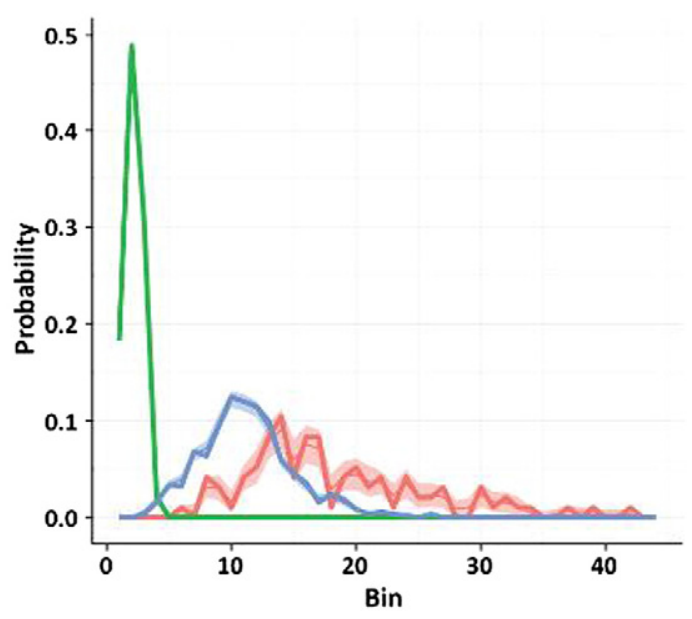

Age $>70$

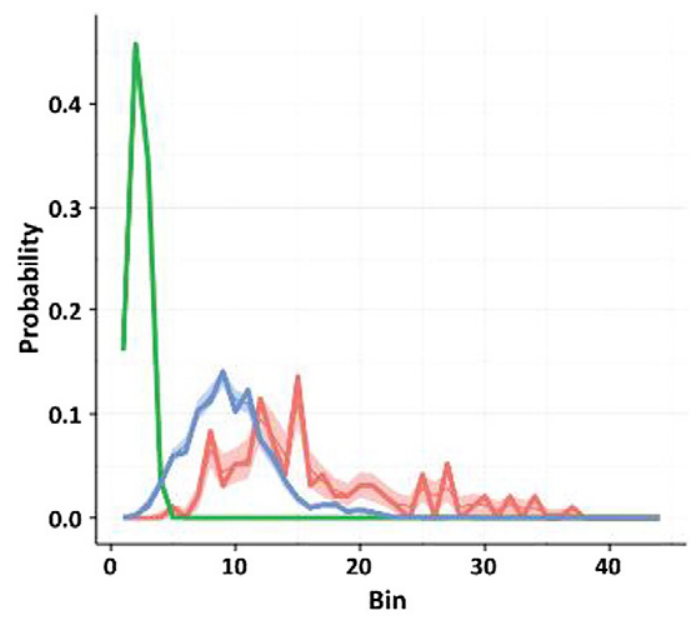

distance 


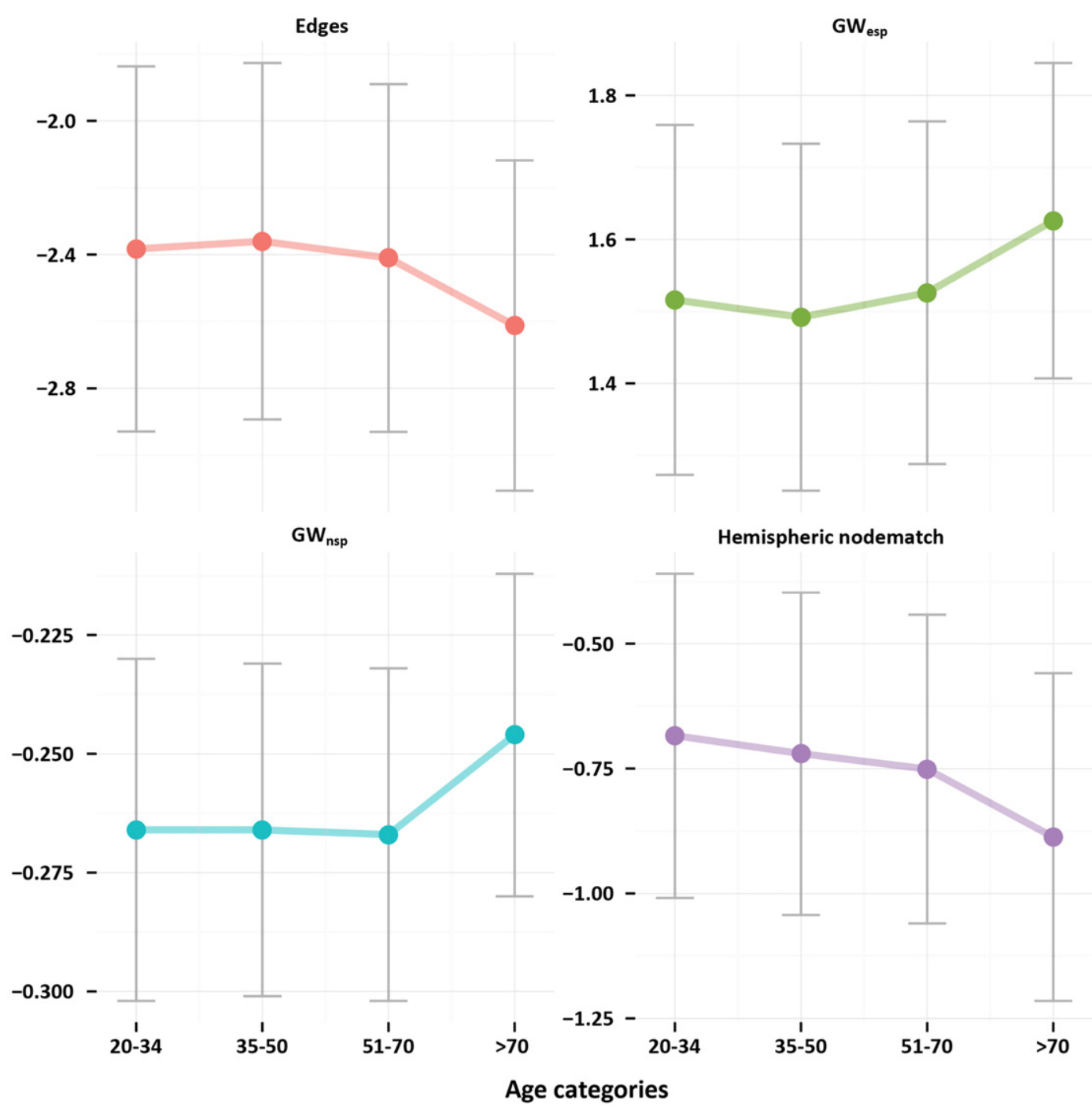

Fig. 5. Network parameters across lifespan. Edges, $\mathrm{GW}_{\mathrm{ESP}}, \mathrm{GW}_{\mathrm{NSP}}$, and hemispheric nodematch for age categories 20-34, 35-50, 51-70, and > 70 years of age.

resulting in density changes. However, the relative contribution of local network structures was stable across age (Fig. 5). Using different thresholds for constructing group based binary networks (Figs. S5 and S6) affected the edges coefficient. This was expected as different thresholds result in different network densities. The edges coefficient captures the density. Different thresholds hardly affected the other coefficients (Fig. S7). Furthermore, using an average of models fitted on ten individual networks, randomly picked from each age category, and resulted in similar coefficients as found with the group-based networks (Fig. S8).

\subsection{Hub-node and random-node damage}

Effects of simulated hub-node lesions on network topology are visualized in Fig. 6. Many connections, mostly in the temporal areas, were lost when $5 \%$ of hub nodes were randomly eliminated. Substantial global loss of connections was evident after elimination of $25 \%$ of hub-nodes, reflecting the abolishing effect of hub-damage on network density.

Quantitative effects of simulated random-node and hub-node lesions, across age categories, are presented in Fig. 7.

With random-node lesions the edges coefficient value decreased with increasing levels of lesioned nodes. A small increasing trend was detected for $\mathrm{GW}_{\mathrm{ESP}}$. For $\mathrm{GW}_{\mathrm{NSP}}$ and hemispheric nodematch parameters, there was no consistent pattern in response to randomnode eliminations.

Hub-node lesions resulted in reduced value of the edges coefficient, with a further decreasing effect as a function of age. GW $\mathrm{GSP}_{\mathrm{E}}$ and $\mathrm{GW}_{\mathrm{NSP}}$ decreased, while hemispheric nodematch increased, after 15-25\% hub lesioning in the higher age categories.

Comparison of vulnerability to lesioning of hub-nodes across lifespan, quantified with Bayes factors between age categories, is given in Table 3. Strongest differences between hub-lesion vulnerability across ages were found for local clustering and hemispheric nodematch. A change in $\mathrm{GW}_{\mathrm{ESP}}$, after $25 \%$ hub damage, was more likely in the age category $>70$ years than in the youngest age category. A change in hemispheric nodematch was more likely in the $>70$ years age group in comparison to the lowest age categories.

\section{Discussion}

We successfully applied Bayesian exponential random graph models on DTI-based structural brain networks over the human lifespan. The exponential random graph modeling approach (Robins et al., 2007a; Snijders et al., 2006) contrasts with conventional graph analysis in being generative instead of descriptive. Furthermore, exponential ran- 


\section{Baseline}

Age 20-34

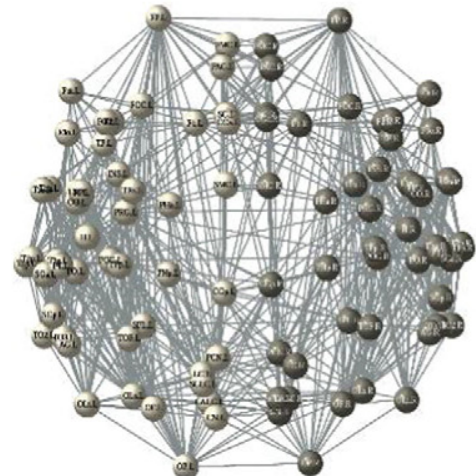

Age 35-50

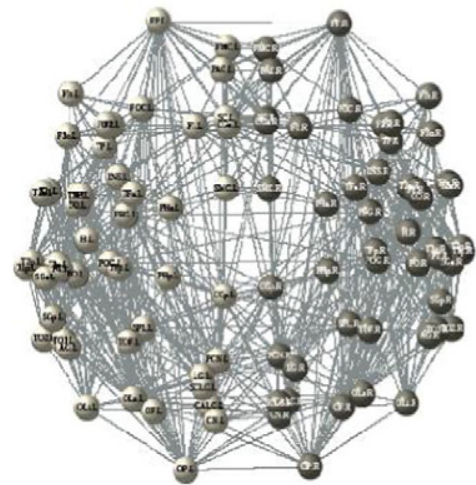

Age 51-70

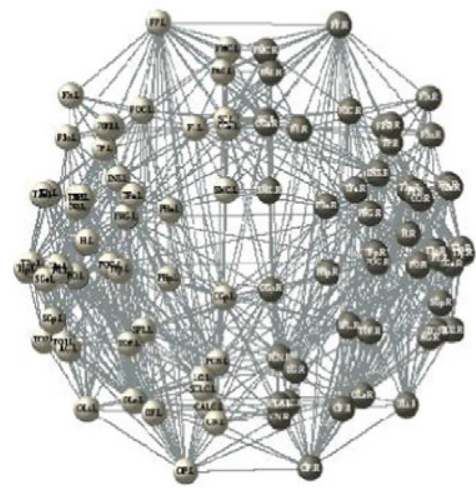

Age $>70$

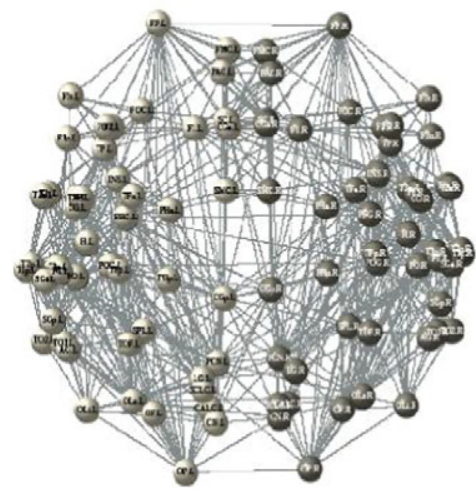

5\% Hub Damage

Age 20-34

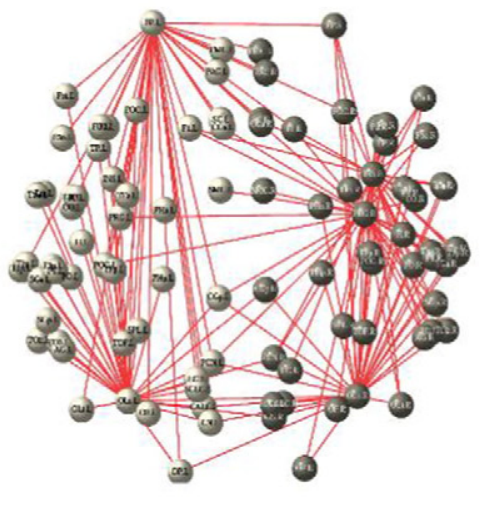

Age 35-50
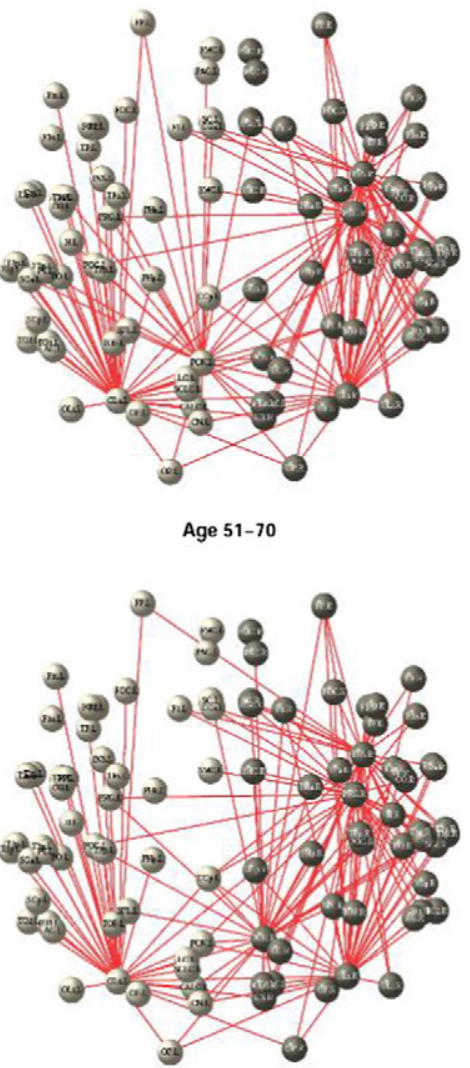

Age $>70$

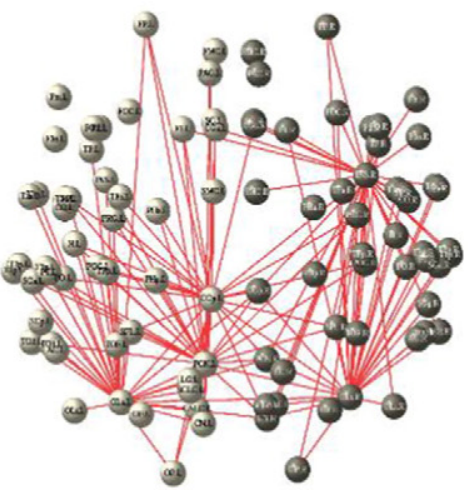

25\% Hub Damage

Age 20-34

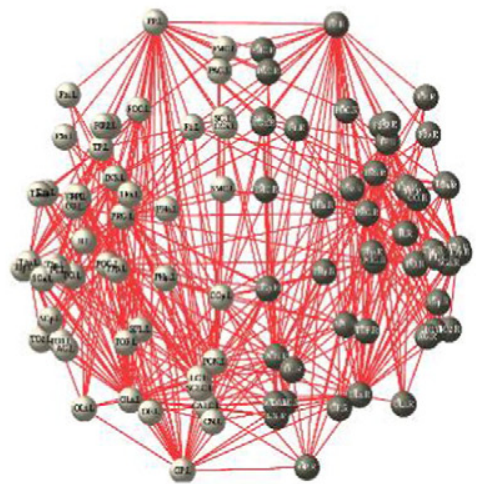

Age 35-50

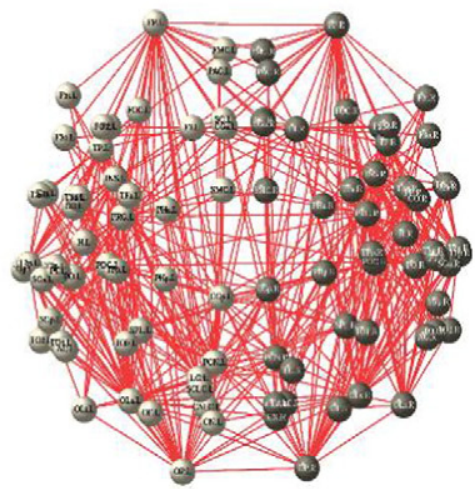

Age 51-70

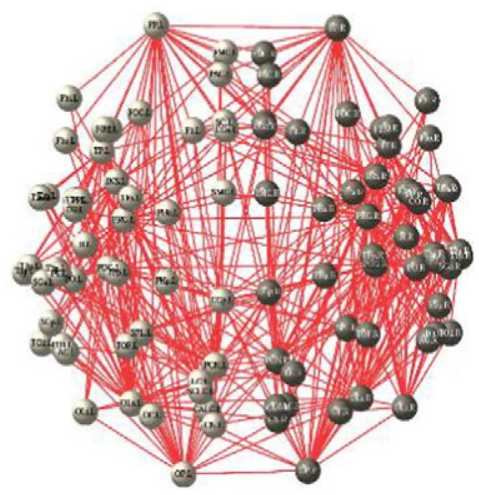

Age $>70$

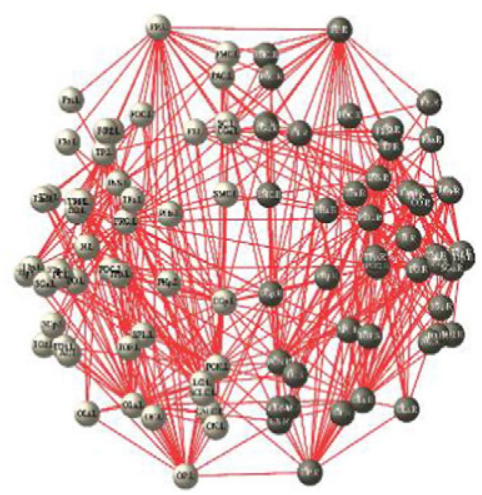


dom graph models do not provide summaries of nodal and global network metrics, but show which local structures contribute to the global network topology and to what extent. Rather than providing solely summary statistics, exponential random graph models allow unbiased characterization of multiple network metric distributions and relational properties in the presence of significant changes in network densities (a problem of descriptive network studies in aging populations) (Caimo and Friel, 2011; Robins et al., 2007a; van Wijk et al., 2010).

In the present study we used a model with three local structures and one node attribute and we showed that simulations including these structures give representative (i.e. well-fitting) networks. The excellent goodness-of-fit of our limited number of local structures suggests that it is possible to describe a complex binary network with a small set of local parameters. We found that all included local structures contributed significantly to the global network topology across the lifespan. The values and signs of the parameters provide information about the relative contribution of local structures to the overall network topology (Robins et al., 2007a, 2007b). The negative coefficients for edges and global efficiency $\left(\mathrm{GW}_{\mathrm{NSP}}\right)$ as well as the positive coefficient for local clustering $\left(\mathrm{GW}_{\mathrm{ESP}}\right)$ are in line with previous studies using the same approach in functional connectivity datasets (Simpson et al., 2011, 2012). The negative edges coefficient imply a lower expected edge density in comparison to a random graph with $50 \%$ edge probability, meaning that the brain network is relatively sparse, which is considered to be economical in terms of wiring costs (Achard and Bullmore, 2007; Bullmore and Sporns, 2012). The positive $\mathrm{GW}_{\mathrm{ESP}}$ reflects the many connections contributing to clustering in the brain. Clustering (and segregation) is a well-known property of the brain contributing to local efficiency and segregation of brain function (Bullmore and Sporns, 2009; Rubinov and Sporns, 2010). The negative values for $\mathrm{GW}_{\mathrm{NSP}}$ imply that nonconnected nodes sharing one or multiple partners (i.e., indirectly connected) are also sparse. In this case we might think of long-range (within- and between-hemisphere) connections that are important for the global efficiency (and integration) of brain function, which is an important feature of brain organization, also in terms of (a trade-off between) wiring costs and efficiency in brain organization (Bullmore and Sporns, 2012). The negative hemispheric nodematch values indicate a tendency

\section{Table 3}

Influence of simulated hub-damage on relative contributions of substructures (edges, $\mathrm{GW}_{\mathrm{ESP}}, \mathrm{GW}_{\mathrm{NSP}}$ and hemispheric nodematch) compared between age categories (20-34 years; $35-50$ years; $51-70$ years; $>70$ years). Difference in the relative contribution of substructures between 0\% (baseline network) and 25\% (hub-lesioned network) hub lesioning is expressed by the $\log$ (Bayes factor).

\begin{tabular}{|c|c|c|c|}
\hline Substructure & $\begin{array}{l}\text { Age category, } \\
\text { baseline network }\end{array}$ & $\begin{array}{l}\text { Age category, } \\
\text { hub-lesioned network }\end{array}$ & $\begin{array}{l}\log \text { (Bayes } \\
\text { factor) }\end{array}$ \\
\hline Edges & $20-34$ & $35-50$ & 2.7 \\
\hline Edges & $20-34$ & $51-70$ & 2.3 \\
\hline Edges & $20-34$ & $>70$ & 2.1 \\
\hline Edges & $35-50$ & $51-70$ & -0.4 \\
\hline Edges & $35-50$ & $>70$ & -0.6 \\
\hline Edges & $51-70$ & $>70$ & -0.2 \\
\hline $\mathrm{GW}_{\mathrm{ESP}}$ & $20-34$ & $35-50$ & -0.2 \\
\hline $\mathrm{GW}_{\mathrm{ESP}}$ & $20-34$ & $51-70$ & 0.0 \\
\hline $\mathrm{GW}_{\mathrm{ESP}}$ & $20-34$ & $>70$ & -0.2 \\
\hline $\mathrm{GW}_{\mathrm{ESP}}$ & $35-50$ & $51-70$ & 0.2 \\
\hline $\mathrm{GW}_{\mathrm{ESP}}$ & $35-50$ & $>70$ & -0.1 \\
\hline $\mathrm{GW}_{\mathrm{ESP}}$ & $51-70$ & $>70$ & -0.3 \\
\hline $\mathrm{GW}_{\mathrm{NSP}}$ & $20-34$ & $35-50$ & 1.9 \\
\hline $\mathrm{GW}_{\mathrm{NSP}}$ & $20-34$ & $51-70$ & -1.7 \\
\hline $\mathrm{GW}_{\mathrm{NSP}}$ & $20-34$ & $>70$ & -0.4 \\
\hline $\mathrm{GW}_{\mathrm{NSP}}$ & $35-50$ & $51-70$ & -3.6 \\
\hline $\mathrm{GW}_{\mathrm{NSP}}$ & $35-50$ & $>70$ & -2.4 \\
\hline $\mathrm{GW}_{\mathrm{NSP}}$ & $51-70$ & $>70$ & 1.3 \\
\hline Hemispheric nodematch & $20-34$ & $35-50$ & 5.1 \\
\hline Hemispheric nodematch & $20-34$ & $51-70$ & 1.9 \\
\hline Hemispheric nodematch & $20-34$ & $>70$ & 3.6 \\
\hline Hemispheric nodematch & $35-50$ & $51-70$ & -3.2 \\
\hline Hemispheric nodematch & $35-50$ & $>70$ & -1.5 \\
\hline Hemispheric nodematch & $51-70$ & $>70$ & 1.7 \\
\hline
\end{tabular}

of nodes to make more connections within hemispheres than between hemispheres, which has been shown by many connectivity studies.

Statistical analyses of exponential random graph models across the lifespan revealed a relatively stable contribution of network properties (i.e. local clustering and global efficiency) over all age categories, in contrast with previous literature showing alterations in both structural (Dennis et al., 2013; Gong et al., 2009; Hagmann et al., 2010; Montembeault et al., 2012; Otte et al., 2015; Wu et al., 2012; Zhu
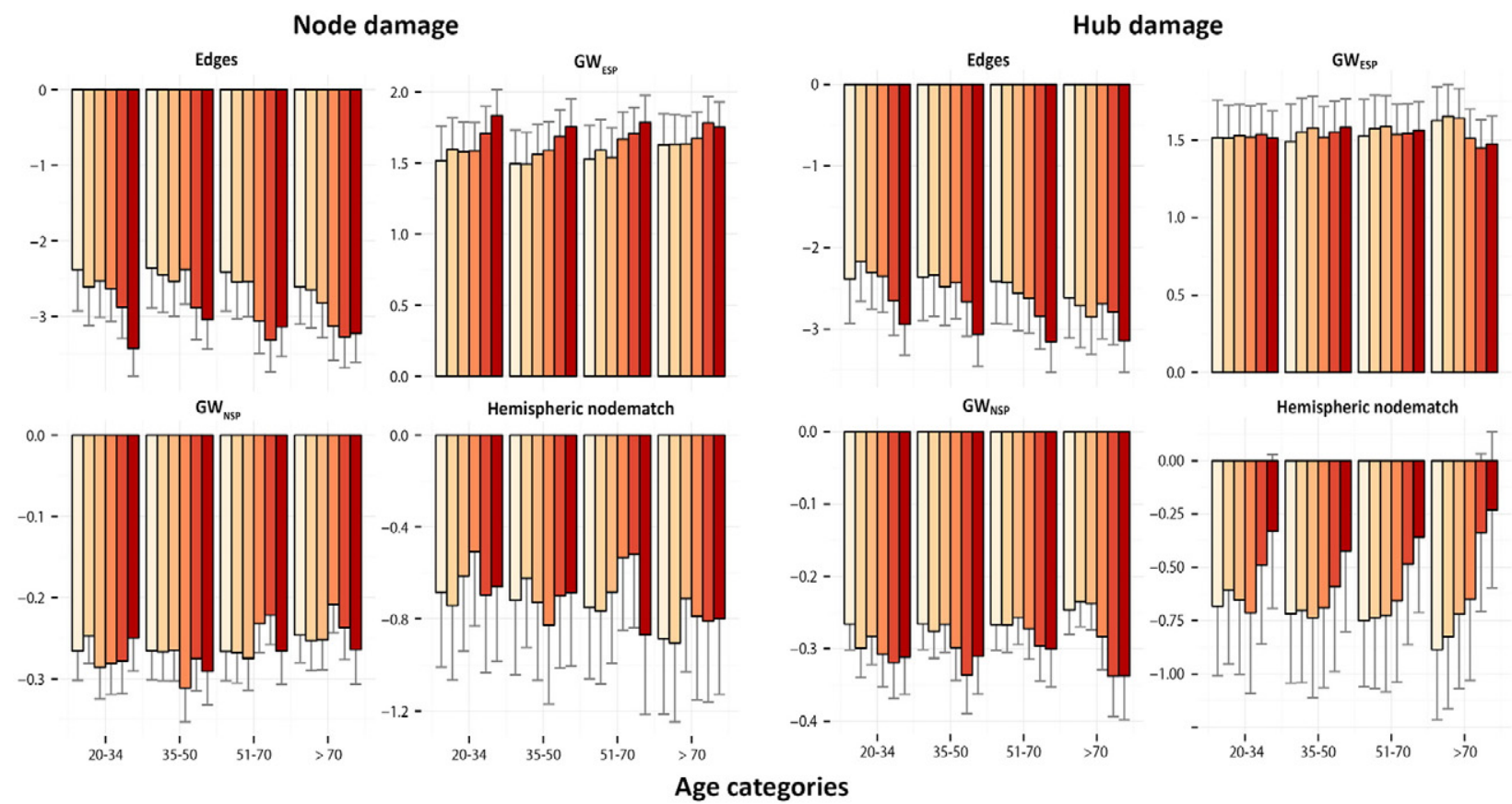

Age categories

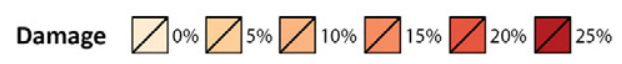

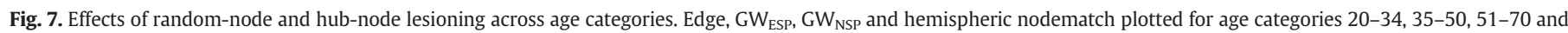
$>70$ years of age, at different percentages (color-coded) of simulated random-node (left) and hub-node (right) lesioning. 
et al., 2012) and functional brain networks across life-span (Achard and Bullmore, 2007; Betzel et al., 2014; Meier et al., 2012; Meunier et al., 2009; Nathan Spreng and Schacter, 2012; Simpson and Laurienti, 2015; Smit et al., 2016; Wang et al., 2012). Furthermore, our findings are also in contrast with significant differences found in a previous exponential random graph modeling study in functional networks (Simpson et al., 2011), and a recently developed similar approach (also discussed below) which revealed differences in functional networks across the lifespan, such as older adults having stronger connections between highly clustered nodes, or less assortativity in visual and multisensory regions (Simpson and Laurienti, 2015). We speculate that functional networks might be more prone to changes across the lifespan, while structural networks remain quite stable, when taking into account density differences and mutual dependencies of network properties.

Simulation of random and hub lesions showed minimal effects on the contribution of local structures (posterior probabilities in favor of a change were between 80 and 90\%) within age categories. This emphasizes the relative robustness to (simulated) brain network damage of local network structures, i.e. unless brain damage local network features that give rise to the global network topology, such as local clustering and global efficiency, are not significantly affected. Nevertheless, hub-node lesioning had a stronger effect in people above 70 years of age as compared to the youngest age category (i.e. $\log$ (Bayes factor) $>3)$. We found trends of increasing local clustering $\left(\mathrm{GW}_{\mathrm{ESP}}\right)$ with more random-node eliminations up to 70 years of age, after which $\mathrm{GW}_{\mathrm{ESP}}$ remained the same or tended to decrease with increasing hub damage. Hub-node lesioning resulted in lower $\mathrm{GW}_{\text {NSP }}$ and higher hemispheric nodematches. These two trends increased with higher age, which may indicate that older people are more vulnerable to hubnode damage (possibly associated with degenerative disease such as Alzheimer's disease (Crossley et al., 2014)) than younger people. This may be due to the overall decrease in network density across age, perhaps in combination with increased vulnerability due to high intrinsic neuronal activity of hub node regions (de Haan et al., 2012). Our findings are in line with previous simulation studies in human and animals, which have shown that computational lesioning of hub regions has stronger effects on structural and functional network topology than lesioning of random nodes (Alstott et al., 2009; Crossley et al., 2014; Kaiser et al., 2007).

There are several other approaches, which are of interest in light of exponential random graph modeling that has been used to capture generative network information. One such approach is based on counting motifs (Sporns and Kötter, 2004), which needs post-hoc statistical assessment, while statistical testing is inherent for exponential random graph models. However, motif counting and corresponding statistical results can provide good starting conditions for choosing appropriate exponential random graph models (van Wijk et al., 2010). In particular because earlier problems such as the intractability of the normalizing constant and model degeneracy have been solved (Caimo and Friel, 2011; Handcock, 2003). Other interesting approaches (complementary with exponential random graph modeling) are mixed models (Simpson et al., 2014) and Gibbs distribution models (La Rosa et al., 2016). In contrast to mixed models, exponential random graph models are limited in examining specific connections and investigating associations between network and phenotypes, and less suited for group comparisons, but are able to capture the inherent dependence structure of complex networks (Simpson et al., 2014). Recently, mixed models were adapted to twopart mixed-effects modeling, to account for the dependence structure (Simpson and Laurienti, 2015, 2016). In contrast to Gibbs distribution models (i.e. modeling of graph populations), both exponential random graph models and mixed models ignore overall network differences, but are able to capture topological features (La Rosa et al., 2016). It would be interesting to adapt and integrate modeling approaches, and if impossible, it is recommended to consider the simultaneous use of complementary approaches in future research.
In line with the earlier mentioned growth models (Betzel et al., 2015; Vertes et al., 2012), exponential random graph models aim for a parsimonious description of the human brain network. Comparison of different generative models with several parameters provided the best model or optimal parameters, resulting in the generation of synthetic networks with very similar topological characteristics as real brain networks (Betzel et al., 2015; Vertes et al., 2012). These studies showed that cost minimization alone does not explain the global network topology, but that the models require information on the pairwise similarity of nodes (i.e. homophily; e.g. similar function or similar degree) to accurately simulate the brain network (Betzel et al., 2015; Vertes et al., 2012). Although modeling a geometrically preferential attachment parameter (i.e. based on distance) is possible with exponential random graph modeling, it requires distance information on all possible node pairs (which is difficult to determine with sparse connectivity data; but Euclidean distance approximations could be used as proxies, with good results). Our study shows that node attributes (e.g. locational information like hemisphere) can be easily implemented in the exponential random graph modeling approach (Caimo and Friel, 2014). It would therefore be interesting to include other attributes in future research (e.g. sensory, motor and association areas) to test for assortativity (i.e. homophily) in terms of functional or organizational similarity (Betzel et al., 2015; Vertes et al., 2012). As such it would also be possible to simulate and compare different models to examine which parameters are of importance to the global network topology.

Recently, another approach, minimum spanning trees, has been proposed to circumvent the methodological issues that arise in the comparison of networks that differ in size and density (Stam et al., 2014). The tree describes the backbone of a network, which contains only the strongest connections, thereby facilitating comparability of different network topologies without aforementioned normalization and correction steps. Several studies have shown the usefulness of the minimum spanning tree approach by detecting subtle conditional and developmental network changes, both between and within studies (Stam et al., 2014). To date, no comparative studies between minimum spanning trees and generative network modeling are known to us. Minimum spanning trees are potentially relevant for modeling of very large (edge-weighted) networks, as exponential random graph models are computationally much more demanding. A drawback of exponential random graph models is the nonlinear increase in computational load with increasing network size and model complexity. Furthermore implementation for edge-valued networks (Desmarais and Cranmer, 2012) are not yet available in the Bayesian framework.

One limitation of the present study might be that subcortical regions were not included. However, the accuracy of registration and alignment of sub-cortical regions is difficult if compared with cortical regions, even when nonlinear registration procedures are used, and might thus give unreliable networks. Another limitation might be the construction of representative group-networks based on a certain, but arbitrary, edge prevalence threshold. Future studies might look into different approaches to fit group networks, e.g. based on exponential random graph modeling (Simpson et al., 2012) or a fully Bayesian description of networks (Hinne et al., 2012; Janssen et al., 2014). The present study did not investigate whether exponential random graph models can solve the problems with differences in network size. This highly interesting and relevant question should therefore be addressed in future research.

Future studies may explore Bayesian generative modeling in other network datasets in health and disease, and extend the model with more substructures and attributes (e.g. cortical regions, assortative connections, brain location, interactions), to assess the effect of different network properties between brain regions (Dennis et al., 2013) as well as constraining influences of network topology and connections (Bullmore and Sporns, 2012; Kaiser and Hilgetag, 2006). 


\section{Contributors}

MRTS and WMO were involved in designing of the study and performed the analysis. All authors contributed to the data interpretation and drafting of the manuscript.

\section{Funding}

This work was supported by the Netherlands Organization for Scientific Research (NWO-VICI 016.130.662, NWO-VENI 016.168.038), and the Dutch Brain Foundation (F2014(1)-06).

\section{Disclosure/conflict of interest}

None of the authors have any conflict of interest to disclose in relation to this work.

\section{Appendix A. Supplementary data}

Supplementary data to this article can be found online at http://dx. doi.org/10.1016/j.neuroimage.2016.04.066.

\section{References}

Achard, S., Bullmore, E., 2007. Efficiency and cost of economical brain functional networks. PLoS Comput. Biol. 3, 0174-0183. http://dx.doi.org/10.1371/journal.pcbi. 0030017.

Alstott, J., Breakspear, M., Hagmann, P., Cammoun, L., Sporns, O., 2009. Modeling the impact of lesions in the human brain. PLoS Comput. Biol. 5. http://dx.doi.org/10.1371/ journal.pcbi.1000408.

Andrews-Hanna, J.R., Snyder, A.Z., Vincent, J.L., Lustig, C., Head, D., Raichle, M.E., Buckner, R.L., 2007. Disruption of large-scale brain systems in advanced aging. Neuron 56 , 924-935. http://dx.doi.org/10.1016/j.neuron.2007.10.038.

Betzel, R.F., Byrge, L., He, Y., Goñi, J., Zuo, X.N., Sporns, O., 2014. Changes in structural and functional connectivity among resting-state networks across the human lifespan. Neurolmage 102, 345-357. http://dx.doi.org/10.1016/j.neuroimage.2014.07.067.

Betzel, R.F., Avena-Koenigsberger, A., Goñi, J., He, Y., de Reus, M.A., Griffa, A., Vértes, P.E., Mišic, B., Thiran, J.-P., Hagmann, P., van den Heuvel, M., Zuo, X.-N., Bullmore, E.T., Sporns, O., 2015. Generative models of the human connectome. NeuroImage 124, 1054-1064. http://dx.doi.org/10.1016/j.neuroimage.2015.09.041.

Bounova, G., De Weck, O., 2012. Overview of metrics and their correlation patterns for multiple-metric topology analysis on heterogeneous graph ensembles. Phys. Rev. E 85, 16117. http://dx.doi.org/10.1103/PhysRevE.85.016117.

Bullmore, E., Sporns, O., 2009. Complex brain networks: graph theoretical analysis of structural and functional systems. Nat. Rev. Neurosci. 10, 186-198. http://dx.doi. org/10.1038/nrn2575.

Bullmore, E., Sporns, O., 2012. The economy of brain network organization. Nat. Rev. Neurosci. 13, 336-349. http://dx.doi.org/10.1038/nrn3214.

Burke, S.N., Barnes, C.A., 2006. Neural plasticity in the ageing brain. Nat. Rev. Neurosci. 7 30-40. http://dx.doi.org/10.1038/nrn1809.

Caimo, A., Friel, N., 2011. Bayesian inference for exponential random graph models. Soc. Networks 33, 41-55. http://dx.doi.org/10.1016/j.socnet.2010.09.004.

Caimo, A., Friel, N., 2014. Bergm: Bayesian exponential random graphs in R. J. Stat. Softw. 61 (2), 1-25. http://dx.doi.org/10.18637/jss.v061.i02.

Caimo, A., Mira, A., 2014. Efficient computational strategies for doubly intractable problems with applications to Bayesian social networks. Stat. Comput. 25, 113-125. http://dx.doi.org/10.1007/s11222-014-9516-7.

Cao, M., Wang, J.H., Dai, Z.J., Cao, X.Y., Jiang, L.L., Fan, F.M., Song, X.W., Xia, M.R., Shu, N., Dong, Q., Milham, M.P., Castellanos, F.X., Zuo, X.N., He, Y., 2014. Topological organization of the human brain functional connectome across the lifespan. Dev. Cogn. Neurosci. 7, 76-93. http://dx.doi.org/10.1016/j.dcn.2013.11.004.

Collier, T.J., Kordower, J.H., 2012. Ageing as a primary risk factor for Parkinson's disease: evidence from studies of non-human primates. Nat. Rev. Neurosci. 12, 359-366. http://dx.doi.org/10.1038/nrn3039.Ageing.

Crossley, N.A., Mechelli, A., Scott, J., Carletti, F., Fox, P.T., Mcguire, P., Bullmore, E.T., 2014. The hubs of the human connectome are generally implicated in the anatomy of brain disorders. Brain 137, 2382-2395. http://dx.doi.org/10.1093/brain/awu132.

de Haan, W., Mott, K., van Straaten, E.C.W., Scheltens, P., Stam, C.J., 2012. Activity dependent degeneration explains hub vulnerability in Alzheimer's disease. PLoS Comput. Biol. 8. http://dx.doi.org/10.1371/journal.pcbi.1002582.

Dennis, E.L., Jahanshad, N., McMahon, K.L., de Zubicaray, G.I., Martin, N.G., Hickie, I.B., Toga, A.W., Wright, M.J., Thompson, P.M., 2013. Development of brain structural connectivity between ages 12 and 30: a 4-tesla diffusion imaging study in 439 adolescents and adults. NeuroImage 64, 671-684. http://dx.doi.org/10.1016/j.neuroimage. 2012.09.004.

Desikan, R.S., Ségonne, F., Fischl, B., Quinn, B.T., Dickerson, B.C., Blacker, D., Buckner, R.L., Dale, A.M., Maguire, R.P., Hyman, B.T., Albert, M.S., Killiany, R.J., 2006. An automated labeling system for subdividing the human cerebral cortex on MRI scans into gyral based regions of interest. NeuroImage 31, 968-980. http://dx.doi.org/10.1016/j. neuroimage.2006.01.021.

Desmarais, B.A., Cranmer, S.J., 2012. Statistical inference for valued-edge networks: the generalized exponential random graph model. PLoS One 7, e30136. http://dx.doi. org/10.1371/journal.pone.0030136.

Fienberg, S.E., Rinaldo, A., Zhou, Y., 2008. On the geometry of discrete exponential families with application to exponential random graph models. Electron. J. Stat. 446-484. http://dx.doi.org/10.1214/08-EJS350.

Fornito, A., Zalesky, A., Breakspear, M., 2013. Graph analysis of the human connectome: promise, progress, and pitfalls. NeuroImage 80, 426-444. http://dx.doi.org/10.1016/ j.neuroimage.2013.04.087.

Frank, O., Strauss, D., 1986. Markov graphs. J. Am. Stat. Assoc. 81, 832-842.

Gong, G., Rosa-Neto, P., Carbonell, F., Chen, Z.J., He, Y., Evans, A.C., 2009. Age- and genderrelated differences in the cortical anatomical network. J. Neurosci. 29, 15684-15693. http://dx.doi.org/10.1523/JNEUROSCI.2308-09.2009.

Goni, J., van den Heuvel, M.P., Avena-Koenigsberger, A., Velez de Mendizabal, N., Betzel, R.F. Griffa, A., Hagmann, P., Corominas-Murtra, B., Thiran, J.-P., Sporns, O., 2014. Restingbrain functional connectivity predicted by analytic measures of network communication. Proc. Natl. Acad. Sci. 111, 833-838. http://dx.doi.org/10.1073/pnas.1315529111.

Hagmann, P., Kurant, M., Gigandet, X., Thiran, P., Wedeen, V.J., Meuli, R., Thiran, J.P., 2007. Mapping human whole-brain structural networks with diffusion MRI. PLoS One 2. http://dx.doi.org/10.1371/journal.pone.0000597.

Hagmann, P., Cammoun, L., Gigandet, X., Meuli, R., Honey, C.J., Van Wedeen, J., Sporns, O., 2008. Mapping the structural core of human cerebral cortex. PLoS Biol. 6, 1479-1493. http://dx.doi.org/10.1371/journal.pbio.0060159.

Hagmann, P., Sporns, O., Madan, N., Cammoun, L., Pienaar, R., Wedeen, V.J., Meuli, R. Thiran, J.-P., Grant, P.E., 2010. White matter maturation reshapes structural connectivity in the late developing human brain. Proc. Natl. Acad. Sci. U. S. A. 107 19067-19072. http://dx.doi.org/10.1073/pnas.1009073107.

Handcock, M.S., 2003. Assessing degeneracy in statistical models of social networks. J. Am. Stat. Assoc. 76, 33-50 doi:10.1.1.81.5086.

Hinne, M., Heskes, T., van Gerven, M.A.J., 2012. Bayesian inference of whole-brain networks $1-10$.

Honey, C.J., Honey, C.J., Sporns, O., Sporns, O., Cammoun, L., Cammoun, L., Gigandet, X. Gigandet, X., Thiran, J.P., Thiran, J.P., Meuli, R., Meuli, R., Hagmann, P., Hagmann, P. 2009. Predicting human resting-state functional connectivity from structural connectivity. Proc. Natl. Acad. Sci. U. S. A. 106, 2035-2040. http://dx.doi.org/10.1073/pnas. 0811168106.

Hunter, D.R., Handcock, M.S., 2006. Inference in curved exponential family models for networks. J. Comput. Graph. Stat. 15, 565-583. http://dx.doi.org/10.1198/ $106186006 \mathrm{X} 133069$.

Hunter, D.R., Handcock, M.S., Butts, C.T., Morris, M., 2009. ERGM: a package to fit, simulate and diagnose exponential-family models for networks. J. Stat. Softw. 24, 1-29.

Janssen, R.J., Hinne, M., Heskes, T., van Gerven, M.A.J., 2014. Quantifying uncertainty in brain network measures using Bayesian connectomics. Front. Comput. Neurosci. 8 , 126. http://dx.doi.org/10.3389/fncom.2014.00126.

Kaiser, M., Hilgetag, C.C., 2006. Nonoptimal component placement, but short processing paths, due to long-distance projections in neural systems. PLoS Comput. Biol. 2, e95. http://dx.doi.org/10.1371/journal.pcbi.0020095.

Kaiser, M., Martin, R., Andras, P., Young, M.P., 2007. Simulation of robustness against lesions of cortical networks. Eur. J. Neurosci. 25, 3185-3192. http://dx.doi.org/10. 1111/j.1460-9568.2007.05574.X.

Klimm, F., Bassett, D.S., Carlson, J.M., Mucha, P.J., 2014. Resolving structural variability in network models and the brain. PLoS Comput. Biol. 10. http://dx.doi.org/10.1371/ journal.pcbi.1003491.

La Rosa, P.S., Brooks, T.L., Deych, E., Shands, B., Prior, F., Larson-Prior, L.J., Shannon, W.D., 2016. Gibbs distribution for statistical analysis of graphical data with a sample application to fcMRI brain images. Stat. Med. 35, 566-580. http://dx.doi.org/10.1002/sim.6757.

Lim, S., Han, C.E., Uhlhaas, P.J., Kaiser, M., 2015. Preferential detachment during human brain development: age- and sex-specific structural connectivity in diffusion tensor imaging (DTI) data. Cereb. Cortex 25, 1477-1489. http://dx.doi.org/10.1093/cercor/bht333.

Meghanathan, N., 2015. Intelligent Systems in Cybernetics and Automation Theory 348, 11-20. http://dx.doi.org/10.1007/978-3-319-18503-3.

Meier, T.B., Desphande, A.S., Vergun, S., Nair, V.a., Song, J., Biswal, B.B., Meyerand, M.E. Birn, R.M., Prabhakaran, V., 2012. Support vector machine classification and characterization of age-related reorganization of functional brain networks. NeuroImage 60, 601-613. http://dx.doi.org/10.1016/j.neuroimage.2011.12.052.

Meunier, D., Achard, S., Morcom, A., Bullmore, E., 2009. Age-related changes in modular organization of human brain functional networks. Neurolmage 44, 715-723. http:// dx.doi.org/10.1016/j.neuroimage.2008.09.062.

Montembeault, M., Joubert, S., Doyon, J., Carrier, J., Gagnon, J.F., Monchi, O., Lungu, O. Belleville, S., Brambati, S.M., 2012. The impact of aging on gray matter structural covariance networks. NeuroImage 63, 754-759. http://dx.doi.org/10.1016/j neuroimage.2012.06.052.

Morris, M., Handcock, M.S., Hunter, D.R., 2008. Specification of exponential-family random graph models. J. Stat. Softw. 24.

Murray, I., Ghahramani, Z., MacKay, D.J.C., 2006. MCMC for doubly-intractable distributions (Citeseer).

Nathan Spreng, R., Schacter, D.L., 2012. Default network modulation and large-scale network interactivity in healthy young and old adults. Cereb. Cortex 22, 2610-2621. http://dx.doi.org/10.1093/cercor/bhr339.

Niccoli, T., Partridge, L., 2012. Ageing as a risk factor for disease. Curr. Biol. 22, R741-R752. http://dx.doi.org/10.1016/j.cub.2012.07.024.

Otte, W.M., van Diessen, E., Paul, S., Ramaswamy, R., Subramanyam Rallabandi, V.P., Stam, C.J., Roy, P.K., 2015. Aging alterations in whole-brain networks during adulthood mapped with the minimum spanning tree indices: the interplay of density, 
connectivity cost and life-time trajectory. NeuroImage 109, 171-189. http://dx.doi. org/10.1016/j.neuroimage.2015.01.011.

Pattison, P., Wasserman, S., 1996. Logit models and logistic regressions for social networks: I. An introduction to Markov graphs and P*. Psychometrika1 61, 401-425. http://dx.doi.org/10.1348/000711099159053.

Raftery, A.E., 1995. Bayesian model selection in social research. Sociol. Methodol. http:// dx.doi.org/10.2307/271063.

Robins, G., Pattison, P., Kalish, Y., Lusher, D., 2007a. An introduction to exponential random graph $\left(\mathrm{p}^{*}\right)$ models for social networks. Soc. Networks 29, 173-191. http://dx. doi.org/10.1016/j.socnet.2006.08.002.

Robins, G., Snijders, T., Wang, P., Handcock, M., Pattison, P., 2007b. Recent developments in exponential random graph $\left(\mathrm{p}^{*}\right)$ models for social networks. Soc. Networks 29 , 192-215. http://dx.doi.org/10.1016/j.socnet.2006.08.003.

Rouder, J.N., Speckman, P.L., Sun, D., Morey, R.D., Iverson, G., 2009. Bayesian t tests for accepting and rejecting the null hypothesis. Psychon. Bull. Rev. 16, 225-237. http:// dx.doi.org/10.3758/PBR.16.2.225.

Rubinov, M., Sporns, O., 2010. Complex network measures of brain connectivity: uses and interpretations. NeuroImage 52, 1059-1069. http://dx.doi.org/10.1016/j.neuroimage. 2009.10.003.

Salat, D.H., 2011. The declining infrastructure of the aging brain. Brain Connect. 1, 279-293. http://dx.doi.org/10.1089/brain.2011.0056.

Simpson, S.L., Laurienti, P.J., 2015. A two-part mixed-effects modeling framework for analyzing whole- brain network data. Neurolmage 113, 310-319.

Simpson, S.L., Laurienti, P.J., 2016. Disentangling brain graphs: a note on the conflation of network and connectivity analyses. Brain Connect. 6, 95-98. http://dx.doi.org/10. 1089/brain.2015.0361.

Simpson, S.L., Hayasaka, S., Laurienti, P.J., 2011. Exponential random graph modeling for complex brain networks. PLoS One 6. http://dx.doi.org/10.1371/journal.pone. 0020039.

Simpson, S.L., Moussa, M.N., Laurienti, P.J., 2012. An exponential random graph modeling approach to creating group-based representative whole-brain connectivity networks NeuroImage 60, 1117-1126. http://dx.doi.org/10.1016/j.neuroimage.2012.01.071.

Simpson, S.L., DuBois Bowman, F., Laurienti, P.J., 2014. NIH Public Access. Stat Surv. 7, 1-36. http://dx.doi.org/10.1016/j.str.2010.08.012.Structure.

Smit, D., De Geus, E., Boersma, M., Boomsma, D., Stam, C., 2016. Life-span development of brain network integration assessed with phase lag index connectivity and minimum spanning tree graphs (Brain Connect)

Snijders, T.A.B., Pattison, P.E., Robins, G.L., Handcock, M.S., 2006. New specifications for exponential random graph models. Sociol. Methodol. 36, 99-153. http://dx.doi.org/10. 1111/j.1467-9531.2006.00176.x.
Sporns, O., 2010. Networks of the brain. MIT Press.

Sporns, O., Kötter, R., 2004. Motifs in brain networks. PLoS Biol. 2, e369. http://dx.doi.org/ 10.1371/journal.pbio.0020369.

Stam, C.J., 2014. Modern network science of neurological disorders. Nat. Rev. Neurosci. 15, 683-695. http://dx.doi.org/10.1038/nrn3801.

Stam, C.J., Tewarie, P., Van Dellen, E., van Straaten, E.C.W., Hillebrand, A., Van Mieghem, P., 2014. The trees and the forest: characterization of complex brain networks with minimum spanning trees. Int. J. Psychophysiol. 92, 129-138. http://dx.doi.org/10.1016/j. ijpsycho.2014.04.001.

Strauss, D., Ikeda, M., 1990. Pseudolikelihood estimation for social networks. J. Am. Stat. Assoc. 85, 204-212. http://dx.doi.org/10.2307/2289546.

Telesford, Q.K., Simpson, S.L., Burdette, J.H., Hayasaka, S., Laurienti, P.J., 2011. The brain as a complex system: using network science as a tool for understanding the brain. Brain Connect. 1, 295-308. http://dx.doi.org/10.1089/brain.2011.0055.

van Wijk, B.C.M., Stam, C.J. Daffertshofer, A., 2010. Comparing brain networks of different size and connectivity density using graph theory. PLoS One 5. http://dx.doi.org/10. 1371/journal.pone.0013701.

Vertes, P.E., Alexander-Bloch, A.F., Gogtay, N., Giedd, J.N., Rapoport, J.L., Bullmore, E.T., 2012. Simple models of human brain functional networks. Proc. Natl. Acad. Sci. 109, 5868-5873. http://dx.doi.org/10.1073/pnas.1111738109.

Wang, R., Benner, T., Sorensen, A.G., Wedeen, V.J., 2007. Diffusion toolkit : a software package for diffusion imaging data processing and tractography. Proc. Int. Soc. Magn. Reson. Med. 15, 3720.

Wang, L., Su, L., Shen, H., Hu, D., 2012. Decoding lifespan changes of the human brain using resting-state functional connectivity MRI. PLoS One 7. http://dx.doi.org/10. 1371/journal.pone.0044530.

Westlye, L.T., Walhovd, K.B., Dale, A.M., Bjørnerud, A., Due-Tønnessen, P., Engvig, A., Grydeland, H., Tamnes, C.K., Østby, Y., Fjell, A.M., 2010. Life-span changes of the human brain white matter: diffusion tensor imaging (DTI) and volumetry. Cereb. Cortex 20, 2055-2068. http://dx.doi.org/10.1093/cercor/bhp280.

Wu, K., Taki, Y., Sato, K., Kinomura, S., Goto, R., Okada, K., Kawashima, R., He, Y., Evans, A.C., Fukuda, H., 2012. Age-related changes in topological organization of structural brain networks in healthy individuals. Hum. Brain Mapp. 33, 552-568. http://dx. doi.org/10.1002/hbm.21232.

Zhu, W., Wen, W., He, Y., Xia, A., Anstey, K.J., Sachdev, P., 2012. Changing topological patterns in normal aging using large-scale structural networks. Neurobiol. Aging 33, 899-913. http://dx.doi.org/10.1016/j.neurobiolaging.2010.06.022. 